\title{
Nonuniform viscosity in the solar nebula and large masses of Jupiter and Saturn
}

\author{
L. Jin ${ }^{\star}$ \\ College of Physics, Jilin University, 119 Jie Fang Rd, Changchun, Jilin 130023, PR China \\ e-mail: jinl@email.unc.edu \\ Dept. of Physics and Astronomy, Clemson Univ., Clemson SC 29634, USA
}

Received 1 June 2004 / Accepted 24 June 2004

\begin{abstract}
I report a novel theory that nonuniform viscous frictional force in the solar nebula accounts for the largest mass of Jupiter and Saturn and their largest amount of $\mathrm{H}$ and He among the planets, two outstanding facts that are unsolved puzzles in our understanding of origin of the Solar System. It is shown that the nebula model of uniform viscosity does not match the present planet masses. By studying current known viscosity mechanisms, I show that viscosity is more efficient in the inner region inside Mercury and the outer region outside Jupiter-Saturn than the intermediate region. The more efficient viscosity drives faster radial inflow of material during the nebula evolution. Because the inflow in the outer region is faster than the intermediate region, the material tends to accumulate in Jupiter-Saturn region which is between the outer and intermediate region. It is demonstrated that the gas trapping time of Jovian planets is longer than the inflow time in the outer region. Therefore the gas already flows to Jupiter-Saturn region before Uranus and Neptune can capture significant gas. But the inflow in the Jupiter-Saturn region is so slow that they can capture large amount of gas before the gas can flow further inward. Hence they have larger masses with larger $\mathrm{H}$ and He content than Uranus and Neptune. I also extend the discussion to the masses of the terrestrial planets, especially low mass of Mercury. The advantages of this theory are discussed.
\end{abstract}

Key words. solar system: formation - solar system: general - planets and satellites: formation - accretion disks

\section{Introduction}

The most widely accepted theory of the origin of the Solar System is the solar nebula hypothesis. Although the theory is, in general, compatible with most of observational facts from the Solar System and star forming regions (Lissauer 1993), a model in which all details are right is not produced yet. Two of the outstanding facts that any theory of the origin of the Solar System must explain are planet masses and their bulk compositions (Lissauer 1993). Jovian planets (Jupiter, Saturn, Uranus, and Neptune) have much larger masses than terrestrial planets (Mercury, Venus, Earth, and Mars). Furthermore Jupiter and Saturn are by far the most massive among the Jovian planets. The terrestrial planets are composed of rocky material while the Jovian planets contain both heavy elements and H, He. The abundance of $\mathrm{H}$ and $\mathrm{He}$ decreases outwards from Jupiter. In this letter, I take the widely used scenario from both the origin of the Solar System and star forming regions. And in that context, I discuss the impact of nonuniform viscosity mechanism on the planet masses and their bulk compositions.

The star formation theory suggests that stars are formed from gravitational collapses of molecular cloud cores.

^ Early ideas on this research began at Clemson Univ. The author is also a visiting scholar at University of North Carolina at Chapel Hill.
Due to the existence of angular momentum, not all material collapses directly into the center to form stars, rather systems of star+disk are formed (the solar nebula is such a disk). It is well known that the internal viscous frictional force in the disk transports the angular momentum outward and the bulk of the disk material diffuses inwards onto the protostar. This naturally explains the angular momentum distribution of the Solar System. This viscous force is often referred to as the angular momentum transport (AMT). Planets are formed in the nebula (Lissauer 1993; Wuchterl et al. 2000). As the material infall slows and ceases, the nebula becomes cool enough that condensates may form. The grains grow via collisions into solid bodies known as planetesimals and sediment towards the midplane of the nebula. These planetesimals then grow into the terrestrial planets and cores of the Jovian planets. The Jovian planets form in the outer part which cools first and is colder than the inner part. Therefore a head start plus ice condensation providing sufficient mass enable the Jovian planets to trap the gases in the nebula before they are dispersed. This accounts for the large masses of the Jovian planets and their abundances of $\mathrm{H}$ and $\mathrm{He}$ while the terrestrial planets are composed of rocky material and have small masses.

Although, based on the current nebula theory and planet formation theory, the interpretation for the difference between 
the Jovian and terrestrial planets is accepted, the reason why Uranus and Neptune have smaller masses and less $\mathrm{H}$ and $\mathrm{He}$ than Jupiter and Saturn is still a puzzle and no theory is widely accepted. 1) The photoevaporation theory (Hollenbach et al. 2000) brings an extra physical process and uncertainties with it, e.g. the assumed photoionization rate exceeds the current solar ultraviolet output by two orders of magnitude. 2) Hydrodynamic accretion theory (Wuchterl et al. 2000; Wuchterl 1995) requires a nebula density that may not be realistic. 3) It is suggested (Wuchterl et al. 2000; Pollack et al. 1996) that the cores of Uranus and Neptune grow more slowly than those of Jupiter and Saturn and do not obtain enough mass to accrete large amount of gas before the gas is dispersed. This scenario requires an effective dispersal process to disperse the gas in Uranus-Neptune region before they accrete large amount of the gas. Such a dispersal process is suggested here. In this letter, I will demonstrate that nonuniform viscous force in the nebula offers a natural solution to this puzzle. I also show advantages of the theory suggested here at the end.

\section{Inconsistency of the nebula model of uniform viscosity with the present planet masses}

The present mass distribution of the planets should give us some idea of the surface density of the nebula. Since the gas is dispersed later, the mass distribution of heavy elements of the planets should reflect the surface density. The well-known minimum mass solar nebula model (Hayash 1981) is constructed as follows: the material in each planet is recovered to the solar composition and spread over an annulus reaching halfway to the orbits of its neighbors. By using the "annulus" approach, the surface density $\Sigma$ from any model can be compared to the masses of the planets. Many nebula models have been built based on a constant $\alpha$ viscosity (uniform AMT) where the viscous stress is scaled with pressure $P$ as $\alpha P$. For example, the similarity solution by Hartmann et al. (1998) shows that $\Sigma$ varies as $\sim r^{-1}$ at small radii and falls sharply at large distances (where $r$ is the heliocentric radius). Notice that $\Sigma$ decreases outward with $r$. I list, in Table 1 (in units of the earth mass, $M_{\oplus}$ ), $M_{\alpha}$, calculated heavy element masses with $\Sigma \sim r^{-1}$ and $M_{\mathrm{h}}$, measured masses of the terrestrial planets or masses of heavy elements of the Jovian planets inferred from current planet model (Guillot 1999). $M_{\alpha}$ is scaled with the heavy element mass of Uranus. From this table, by comparing heavy element masses of the planets with those obtained from $\Sigma \sim r^{-1}$, I discover that the nebula model of constant $\alpha$ (uniform AMT) does not match the planet masses and Jupiter and Saturn masses are significantly enhanced. Notice that by "the mass enhancement" throughout this letter, I mean the enhancement compared with the nebula model of constant $\alpha$. The terrestrial planets also have some enhancement except the famous Mars drop and low Mercury mass. I will discuss these together later.

\section{Viscosity values in different regions of the Nebula}

I use the widely accepted approach for $\alpha$ values (Papaloizou \& Lin 1995; Stone et al. 2000; Balbus 2003). For the case of
Table 1. Planet masses of heavy elements.

\begin{tabular}{crr}
\hline \hline Planet & $M_{\mathrm{h}}\left(M_{\oplus}\right)$ & $M_{\alpha}\left(M_{\oplus}\right)$ \\
\hline Mercury & 0.055 & 0.4 \\
Venus & 0.81 & 0.37 \\
Earth & 1.0 & 0.48 \\
Mars & 0.11 & $2.6(0.30)$ \\
Jupiter & $11-42$ & $4.9(7.1)$ \\
Saturn & $19-31$ & 8.4 \\
Uranus & 12.5 & 12.5 \\
Neptune & 15 & 12 \\
\hline
\end{tabular}

the solar nebula, see the review by Stone et al. (2000) and references therein. It seems that hydrodynamic turbulence is ineffective as an AMT mechanism. Gravitational instability can transport angular momentum when the nebula is massive (Laughlin \& Bodenheimer 1994; Papaloizou \& Lin 1995). The effective value of $\alpha$ is $\sim 0.03-0.1$. This can dominate the AMT during the early stage. Much of the stellar mass may be gained this way. As the nebula mass drops, less efficient AMT processes take over. The MHD (magnetohydrodynamic) turbulence driven by the magnetorotational instability (MRI) is a very likely mechanism (Stone et al. 2000). The viscosity is high (low) when the MRI can (not) survive. The ideal MHD simulations give values of $\alpha$ ranging from $5 \times 10^{-3}$ to $\sim 0.6$. The high value is reached when there is a net vertical field. A typical value for $\alpha$ is $10^{-2}$. The solar magnetic field may provide such a net vertical field inside Mercury if the solar dynamo starts that early. Wave propagation alone is a less effective AMT than the MHD turbulence. The excitation is most powerful in the outer region of the nebula. This may favor high $\alpha$ in the outer region. The value used to fit observations of accretion rates (Hartmann et al. 1998) is $\alpha \sim 10^{-2}$. The age consideration also indicates $\alpha \sim 10^{-3}-10^{-2}$.

Jin (1996) considered the effect of ohmic diffusion on the MRI and showed that the MRI is damped when the diffusion rate is greater than the MRI growth rate. The diffusion rate is high (low) when the ionization degree is low (high). The MRI can survive at temperature $T>1000 \mathrm{~K}$ due to the thermal ionization. This temperature can be reached in the inner region of the nebula inside Mercury. Cosmic rays can partially ionize the part of the nebula where they can penetrate (Hayash 1981). So the cosmic ray ionization is more significant where $\Sigma$ is low, which is the outer region of the nebula. Thus the MRI can survive there. An estimate of the location of the transition zone between the outer region and the intermediate region (where the MRI can not survive) can be found by equating the cosmic ray penetration depth $\Sigma_{\mathrm{CR}}=100 \mathrm{~g} \mathrm{~cm}^{-2}$ with $\Sigma$. For an easy estimate, I use Hayashi (1981) surface density and find that the transition zone is around $r \sim 7 \mathrm{AU}$, which is Jupiter-Saturn region. Notice that this radius is larger when the nebula has more mass than the minimum mass. MHD simulations (Fleming \& Stone 2003) finds that the viscosity can drop below $\alpha \sim 10^{-4}$ where $\Sigma$ is large. To summarize, the MHD turbulence driven by the MRI causes high $\alpha\left(\sim 10^{-2}\right)$ in the inner region inside Mercury and the outer region outside Jupiter-Saturn, and $\alpha$ 




Fig. 1. A top view of the solar nebula illustrating the various regions of the viscosity. Inner region: MRI (magnetorotational instability) survives due to the thermal ionization. High viscosity, high radial inflow velocity, and short inflow time. Intermediate region: MRI is suppressed. Low viscosity, low inflow velocity, and long inflow time. Outer region: MRI survives due to the ionization by cosmic rays. High viscosity, high inflow velocity, and short inflow time. The material tends to accumulate in the Jupiter-Saturn region due to the difference of the inflow velocity between the intermediate and outer regions. The gas inflow time in Jupiter-Saturn region is long enough that they have time to capture the gas.

$\left(\sim 10^{-4}\right)$ is significantly lower in the intermediate region due to the damping of the MRI (Fig. 1).

\section{Mass accumulation and gas trapping of Jupiter and Saturn}

Lets look at the mass enhancement in Jupiter-Saturn region due to the above nonuniform $\alpha$ (AMT). The radial inflow velocity is (Pringle 1981)

$v_{r} \approx-\frac{v}{r}=-\alpha c_{\mathrm{s}} \frac{H}{r}=-\alpha\left(\frac{H}{r}\right)^{2} r \Omega$,

where $v$ is the kinematic viscosity, $c_{\mathrm{s}}$ is the sound speed, $H$ is the nebula thickness, and $\Omega$ is the angular velocity. The negative sign means that the material flows inwards to the Sun. Notice that the more efficient AMT drives faster radial inflow. Therefore the inflow in the outer region is faster than the intermediate region and the material tends to accumulate in the transition zone (Fig. 1). This means that Jupiter and Saturn have access to more material. Mathematically, the mass enhancement in Jupiter-Saturn region can be shown as the following. The mass inflow rate at any radius $r$ is (Pringle 1981)

$\dot{M}=-2 \pi r \Sigma v_{r}$
Let $\Sigma_{0}(r)$ be the solution of the surface density with constant $\alpha=\alpha_{\text {o }}$ (the $\alpha$ value in the outer region). Assuming that $\Sigma_{0}$ is not changed for the first order, the mass enhancement rate in the transition zone compared with constant $\alpha$ solution is

$$
\begin{aligned}
\Delta \dot{M}= & \left(\left.2 \pi r \Sigma_{0} v_{r}\right|_{r=R_{\mathrm{i}}}-\left.2 \pi r \Sigma_{0} v_{r}\right|_{r=R_{\mathrm{o}}}\right)_{\alpha=\alpha_{\mathrm{n}}} \\
& -\left(\left.2 \pi r \Sigma_{0} v_{r}\right|_{r=R_{\mathrm{i}}}-\left.2 \pi r \Sigma_{0} v_{r}\right|_{r=R_{\mathrm{o}}}\right)_{\alpha=\alpha_{\mathrm{o}}},
\end{aligned}
$$

where $R_{\mathrm{i}}$ and $R_{\mathrm{O}}$ are the inner and outer radii of the transition zone, and $\alpha_{\mathrm{n}}$ is the nonuniform $\alpha$. The term in first parenthesis is the mass change rate in the zone with nonuniform $\alpha$ and the second is the rate with constant $\alpha=\alpha_{\mathrm{o}}$. Since $\alpha_{\mathrm{n}}=\alpha_{\mathrm{o}}$ at $r=R_{\mathrm{o}}$, two terms at $R_{\mathrm{o}}$ are cancelled. We have by using Eq. (1)

$\Delta \dot{M}=\left.2 \pi r \Sigma_{0}\left(\alpha_{\mathrm{o}}-\alpha_{\mathrm{i}}\right)\left(\frac{H}{r}\right)^{2} r \Omega\right|_{r=R_{\mathrm{i}}}$,

where $\alpha_{\mathrm{i}}$ is the $\alpha$ value in the intermediate region. It is straightforward to see that there is a mass enhancement in the transition zone which is Jupiter-Saturn region since $\alpha_{\mathrm{i}}$ is significantly lower than $\alpha_{\mathrm{o}}$. The enhancement is due to the difference in the radial velocity caused by the difference of $\alpha$ value. If the nebula ever reaches a quasi-steady state ( $\dot{M}$ does not change with $r$ ), Eq. (2) gives that $\Sigma$ is higher in the lower $\alpha$ region which is the intermediate region.

In the process of the planet formation, grains are decoupled from the gas when they grow into larger solid bodies. The inflow keeps the same initial solar composition before the decoupling. I suggest that the mass enhancement in Jupiter-Saturn region before the decoupling explains their enhancement of heavy element masses (Table 1). After the decoupling, the gas will continue its inflow. The inflow time is (Pringle 1981)

$t_{v} \approx-\frac{r}{v_{r}} \approx \alpha^{-1}\left(\frac{H}{r}\right)^{-2} \Omega^{-1}=72 \alpha^{-1}\left(\frac{r}{\mathrm{AU}}\right) \mathrm{yr}$,

where the value of $H / r$ from Hayashi (1981) is used. I list $\alpha$ and calculated inflow times at the heliocentric radius of the planets in Table 2. After formation of solid cores, the first phase of the gas trapping of giant planets is the slow capture of the surrounding hydrogen-helium which takes a few $10^{6} \mathrm{yr}$. Then a rapid accretion of the gas only takes $\sim 10^{5} \mathrm{yr}$ (Pollack et al. 1996). By comparing the slow capture time with the inflow time (Table 2), the main discovery of this letter is the following. The inflow is faster than the capture for Uranus and Neptune and slower for Jupiter and Saturn. Therefore before Uranus and Neptune can capture enough gas to reach the rapid accretion, the gas already flows to Jupiter-Saturn region. This explains the low masses and low $\mathrm{H}-\mathrm{He}$ abundances of Uranus and Neptune among the Jovian planets. But Jupiter and Saturn have enough time to capture the gas before the gas can flow further inward so that they have large masses and large $\mathrm{H}-\mathrm{He}$ abundances (Fig. 1). The fact that Jupiter has bigger mass than Saturn is rarely addressed. I suggest that because Jupiter region has lower $\alpha$ (the higher $\Sigma$, the less cosmic ray penetration) than Saturn, Jupiter has more time to trap gas. 
Table 2. Inflow time (in year) at the heliocentric radius $r$ of the planets.

\begin{tabular}{crrc}
\hline \hline Planet & $r(\mathrm{AU})$ & $\alpha$ & $t_{v}(\mathrm{yr})$ \\
\hline Mercury & 0.39 & $10^{-2}$ & $3 \times 10^{3}$ \\
Venus & 0.72 & $10^{-4}$ & $5 \times 10^{5}$ \\
Earth & 1.0 & $10^{-4}$ & $7 \times 10^{5}$ \\
Mars & 1.5 & $10^{-4}$ & $1 \times 10^{6}$ \\
Jupiter & 5.2 & $10^{-4}$ & $4 \times 10^{6}$ \\
Saturn & 9.5 & $10^{-4}$ & $7 \times 10^{6}$ \\
Uranus & 19 & $10^{-2}$ & $1 \times 10^{5}$ \\
Neptune & 30 & $10^{-2}$ & $2 \times 10^{5}$ \\
\hline
\end{tabular}

\section{Application to other planets and discussion}

Mars is the terrestrial planet adjacent to the Jovian planets. The famous Mars drop (Table 1) might be caused by the sweeping of early-formed Jupiter. If the sweeping extends to Mars orbit, calculated masses for Jupiter and Mars with $\Sigma \sim r^{-1}$ are put in parenthesis in Table 1. The match with observed masses are much better for Mars. The mass enhancement of Earth and Venus (Table 1) is due to the low viscosity (slow inflow). AMT is very efficient in the inner region inside Mercury because of MHD turbulence and it might be even enhanced due to the possible net flux of the solar magnetic field. In addition, magnetic winds may contribute to AMT (Papaloizou \& Lin 1995). Contrary to Jupiter-Saturn region, the material tends to deplete in Mercury region because the inflow is faster in the inner region than in the intermediate region (Fig. 1). So this theory also provides a natural interpretation of the low mass of Mercury (Table 1). Notice that this theory does not contradicts theory of dynamics of planetesimals.

Some of advantages of the new theory presented in this letter are: 1) it is simple and comes natural because it is, without any additional contrived assumptions or physical processes (therefore no new uncertainties), based on the well known wisdom that there is AMT during the nebula evolution in order to understand the current distribution of the angular momentum of the Solar System. The mass distribution due to the AMT is inevitable. 2) The theory uses only one physical mechanism, AMT, to give an unified picture of the planet masses and compositions. In addition to the large masses of Jupiter and Saturn and their large amount of $\mathrm{H}$ and $\mathrm{He}$, the theory might explain low mass of Mercury, the difference between Saturn and Jupiter, and the enhancement of heavy elements of Saturn, Jupiter, Earth, and Venus relative to the model with constant $\alpha$. These facts are rarely addressed. No previous theories have put all of these together. 3) The interpretation for the difference between Jupiter-Saturn and Uranus-Neptune does not depend on the details of the formation processes of the cores of the Jovian planets. The mass redistribution due to the nonuniform AMT is independent of details of planet formation. This theory does not in any way contradicts existing models of planet formation. This would make the theory more viable. Although researchers can have different interpretations for planet masses, it seems clear to the author that the impact of the nonuniform AMT on the planet masses can not be ignored. Notice that the results of this letter depend only on the final values of viscosity, but not on details of AMT, such as what drives AMT.

Acknowledgements. I thank Donald Clayton for reading and comments on the present work and Jin Wang for suggestions of revising the manuscript.

\section{References}

Balbus, S. 2003, ARA\&A, 41, 555

Fleming, T., \& Stone, J. 2003, ApJ, 585, 908

Guillot, T. 1999, Science, 286, 72

Hartmann, L., Calvet, N., Gullbring, E., \& D’Alessio, P. 1998, ApJ, 495,385

Hayashi, C. 1981, Prog. Theor. Phys. Suppl., 70, 35

Hollenbach, D., Yorke, H., \& Johnstone, D. 2000, in Protostars and Planets IV, ed. V. Mannings, A. Boss, \& S. Russell (Tucson: Univ. Arizona Press), 401

Jin, L. 1996, ApJ, 457, 798

Laughlin, G., \& Bodenheimer, P. 1994, ApJ, 436, 335

Lissauer, J. 1993, ARA\&A, 31, 129

Papaloizou, J., \& Lin, D. 1995, ARA\&A, 33, 505

Pollack, J., Hubickyj, O., Bodenheimer, P., et al. 1996, Icarus, 124, 62 Pringle, J. 1981, ARA\&A, 19, 137

Stone, J., Gammie, C., Balbus, S., \& Hawley, J. 2000, in Protostars and Planets IV, ed. V. Mannings, A. Boss, \& S. Russell (Tucson: Univ. Arizona Press), 589

Wuchterl, G. 1995, Earth Moon Planets, 67, 51

Wuchterl, G., Guillot, T., \& Lissauer, J. 2000, in Protostars and Planets IV, ed. V. Mannings, A. Boss, \& S. Russell (Tucson: Univ. Arizona Press), 1081 